\title{
Erratum to: Database preference queries - a possibilistic logic approach with symbolic priorities
}

\author{
Didier Dubois • Allel Hadjali • Henri Prade • \\ Fayçal Touazi
}

Published online: 27 February 2015

(C) Springer International Publishing Switzerland 2015

\begin{abstract}
This note corrects a claim made in the above-mentioned paper about the exact representation of a conditional preference network by means of a possibilistic logic base with partially ordered symbolic weights. We provide a counter-example that shows that the possibilistic logic representation is indeed not always exact. This is the basis of a short discussion on the difficulty of obtaining an exact representation.
\end{abstract}

This note corrects a claim made in [6] about the representation of Conditional Preference networks (CP-nets for short) [1] by means of a possibilistic logic base [2], as well as a similar claim in $[7,8]$.

A CP-net encodes a set of preference statements concerning the values of Boolean decision variables, conditioned on the values of other Boolean decision variables that influence the former. More formally, let $V=\left\{X_{1}, \cdots, X_{n}\right\}$ be a set of Boolean variables. We denote by $\operatorname{Ast}(S)$ the set of interpretations of variables of $S(\subseteq V)$.

Definition 1 A CP-net $\mathcal{N}$ over $V=\left\{X_{1}, \cdots, X_{n}\right\}$ is a directed graph with nodes $X_{1}, \cdots, X_{n}$, and there is a directed edge from $X_{i}$ to $X_{j}$ if the preference about the value $X_{j}$ depends on the value of $X_{i}$. Each node $X_{i} \in V$ is associated with a conditional preference table $C P T_{i}$ that associates a strict preference $\left(x_{i}>\neg x_{i}\right.$ or $\left.\neg x_{i}>x_{i}\right)$ with each

The online version of the original article can be found at http://dx.doi.org/10.1007/s10472-012-9279-9

\footnotetext{
A. Hadjali

IRISA/ENSSAT, Université Rennes I, 6 rue de Kérampont, 22305 Lannion Cedex, France e-mail: hadjali@enssat.fr

D. Dubois $\cdot$ H. Prade $(\bowtie) \cdot$ F. Touazi

IRIT, Université Paul Sabatier, 118 route de Narbonne, 31062 Toulouse Cedex 9, France e-mail: prade@irit.fr

D. Dubois

e-mail: dubois@irit.fr

F. Touazi

e-mail: faycal.touazi@irit.fr
} 
possible instantiation $u_{i}^{j} \in A s t\left(P a\left(X_{i}\right)\right)$ of the parents $P a\left(X_{i}\right)$ of $X_{i}$ (if any). Each entry in a conditional preference table $C P T_{i}$ is of the form $\phi=u_{i}^{j}: \star_{j} x_{i}>\star_{j} \neg x_{i}$, where $u_{i}^{j} \in \operatorname{Ast}\left(\operatorname{Pa}\left(X_{i}\right)\right), \star_{j}$ is blank if the preference is $x_{i}>\neg x_{i}$ and is $\neg$ otherwise.

Each interpretation $\omega$, i.e., an instantiation of all variables in $V$, is understood as a solution to the decision problem described by the preference statements in the CP-net. A CP-net induces a partial preference ordering over solutions defined as follows. Each conditional preference statement $u_{i}^{j}: \star_{j} x_{i}>\star_{j} \neg x_{i}$ expresses a preference between any two solutions $\omega_{1}$ and $\omega_{2}$ that satisfy $u_{i}^{j}$ and only differ on variable $x_{i}$, that is, $\omega_{1}$ satisfies $x_{i}$ and $\omega_{2}$ satisfies $\neg x_{i}$. Namely, the preference of $x_{i}$ over $\neg x_{i}$ is valid in context $u_{i}^{j}$, all other things being equal, what is called the ceteris paribus assumption.

Definition 2 A worsening flip consists in turning an interpretation $\omega_{1}$ into $\omega_{2}$ by flipping the truth-value of a single variable $x_{i}$, so that $\omega_{1}$ is preferred to $\omega_{2}$.

In other words, a worsening flip compares two solutions differing only on one variable, according to the conditional preference table of this variable, completed by applying a ceteris paribus principle to the variables that do not appear in the table.

Definition 3 A CP-net $\mathcal{N}$ defines a partial order $\succ_{\mathcal{N}}$ over the interpretations of $V=$ $\left\{X_{1}, \cdots, X_{n}\right\}$ such that $\omega_{1} \succ_{\mathcal{N}} \omega_{2}$ if and only if there is a sequence of worsening flips changing $\omega_{1}$ into $\omega_{2}$.

The encoding of a CP-net in possibilistic logic is supposed to be made as follows [2, 6]:

- Each entry of the form $u_{i}^{j}: \star_{j} x_{i}>\star_{j} \neg x_{i}$ in the table $C P T_{i}$ for each node $X_{i}, i=$ $1, \ldots, n$ is encoded by the possibilistic logic clause $\left(\neg u_{i}^{j} \vee \star_{j} x_{i}, \alpha_{i}\right)$, where $\alpha_{i}$ is a symbolic weight (whose value is unspecified). This is the syntactic counterpart of the constraint $N\left(\neg u_{i}^{j} \vee \star_{j} x_{i}\right) \geq \alpha_{i}>0$, where $N$ is a necessity measure [3], $\alpha_{i}$ is a symbolic weight representing a certainty value in a necessity scale (a totally ordered set with bottom element 0 ).

- Since the same weight is attached to each clause built from $C P T_{i}$, the set of weighted clauses induced from $C P T_{i}$ is equivalent to one weighted formula $\left(\phi_{i}, \alpha_{i}\right)$, for each variable $X_{i}$, where $\phi_{i}=\bigwedge_{u_{i}^{j} \in \operatorname{Ast}\left(P a\left(X_{i}\right)\right)} \neg u_{i}^{j} \vee \star_{j} x_{i}$, since $N(\phi \wedge \psi)=$ $\min (N(\phi), N(\psi))$. So, each node in the CP-net is associated with a single possibilistic pair made of a propositional logic formula and a symbolic weight.

- Additional constraints over symbolic weights are added. The weight $\alpha_{i}$ attached to each node $X_{i}$, is supposed to be strictly smaller than the weight of each of its parents (thus accounting for the observed priority of father nodes over children nodes in CP-nets).

Let $\Sigma_{\mathcal{N}}$ be the possibilistic base that encodes the CP-net $\mathcal{N}$. At the semantic level, we can associate to each interpretation $\omega$ of the propositional language generated by $V=$ $\left\{X_{1}, \cdots, X_{n}\right\}$ a vector $\omega\left(\Sigma_{\mathcal{N}}\right)$ with as many components as formulas in $\Sigma_{\mathcal{N}}$. In the $i^{t h}$ component of the vector $\omega\left(\Sigma_{\mathcal{N}}\right)$ associated to the weighted formula $\left(\phi_{i}, \alpha_{i}\right) \in \Sigma_{\mathcal{N}}$, we put 1 if $\omega$ satisfies $\phi_{i}$ and $1-\alpha_{i}$ if not, in agreement with possibilistic logic semantics [3]. Here, $1-($.$) just denotes the order-reversing map of the necessity scale, so that 1>1-\alpha_{i}, \forall i$ (due to $\left.\alpha_{i}>0\right)$. Vectors $\omega\left(\Sigma_{\mathcal{N}}\right)$ associated with each interpretation $\omega$, have a specific format. Namely their component $v_{i}$ (one per CP-net node) lies in $\left\{1,1-\alpha_{i}\right\}$ for $i=1, \ldots, n$. The 
comparison between these vectors is solely dictated by the constraints relating the weights of father and children nodes together with the assumption $1>1-\alpha_{i}, \forall i$.

Let $\mathrm{v}$ and $\mathrm{v}^{\prime}$ be two vectors having the same number $n$ of components that lie in a partially ordered set. Let $C(\mathrm{v})=\left\{v_{i}: i=1, \ldots n\right\}$ be the set of distinct components appearing in $\mathrm{v}$, and $\min C(\mathrm{v})$ denote the set of least elements in $C(\mathrm{v})$. Here, the components of the vectors $\omega\left(\Sigma_{\mathcal{N}}\right)$ lie in the set $\left\{1,1-\alpha_{i}: i=1, \ldots, n\right\}$, where the $\alpha_{i}$ 's are distinct and positive. These vectors can then be compared by means of one of the following ordering relations.

Definition $4(\min ) \mathrm{v} \succ_{\min } \mathrm{v}^{\prime}$ iff $\min \left(C(\mathrm{v}) \cup C\left(\mathrm{v}^{\prime}\right)\right) \subseteq C\left(\mathrm{v}^{\prime}\right)$.

This is the usual ordering on interpretations induced by a possibilistic knowledge base, here extended to partial orders. It can be refined as follows:

Definition 5 (discrimin) Delete all pairs $\left(v_{i}, v_{i}^{\prime}\right)$ such that $v_{i}=v_{i}^{\prime}$ in $\mathrm{v}$ and $\mathrm{v}^{\prime}$. Thus, we get two sets $R(\mathrm{v})$ and $R\left(\mathrm{v}^{\prime}\right)$ of remaining components. Then, $\mathrm{v} \succ_{\text {discrimin }} \mathrm{v}^{\prime}$ iff $\min (R(\mathrm{v}) \cup$ $\left.R\left(\mathrm{v}^{\prime}\right)\right) \subseteq R\left(\mathrm{v}^{\prime}\right)$.

This partial ordering refines the previous one and can be further refined as follows:

Definition 6 (leximin) Let $\mathrm{v}^{\sigma}$ be the reordered vector $\mathrm{v}$ by permutation $\sigma$ of its components, i.e., $\mathrm{v}_{i}^{\sigma}=\mathrm{v}_{\sigma(i)}$. Then $\mathrm{v} \succ_{\text {leximin }} \mathrm{v}^{\prime}$ iff $\exists \sigma, \mathrm{v}^{\sigma} \succ_{\text {discrimin }} \mathrm{v}^{\prime}$.

The leximin comparison comes down to deleting all pairs $\left(v_{i}, v_{j}^{\prime}\right)$ such that $v_{i}=v_{j}^{\prime}$ in $\mathrm{v}$ and $\mathrm{v}^{\prime}$ (each deleted component can be used only one time in the deletion process). Thus, we get two minimal non overlapping sets $R^{*}(\mathrm{v})$ and $R^{*}\left(\mathrm{v}^{\prime}\right)$ of remaining components, namely $R^{*}(\mathrm{v}) \cap R^{*}\left(\mathrm{v}^{\prime}\right)=\emptyset$. Then, the leximin ordering comes down to $v \succ_{\text {leximin }} v^{\prime}$ iff $\min \left(R^{*}(\mathrm{v}) \cup R^{*}\left(\mathrm{v}^{\prime}\right)\right) \subseteq R^{*}\left(\mathrm{v}^{\prime}\right)$. As shown in [5], for vectors of the form $\omega\left(\Sigma_{\mathcal{N}}\right)$, the discrimin and leximin orderings coincide, because the coefficients $1-\alpha_{i}$ in different components always differ.

It was claimed in [6] that the above possibilistic logic encoding of a CP-net can exactly capture the CP-net ordering (defined in terms of worsening flips, as recalled at the beginning of this note) using the leximin (or discrimin) order for comparing vectors associated to interpretations of the corresponding possibilistic base. Indeed, each vector reflects the preference constraints that are satisfied or are violated by the considered solution. Unfortunately, this is true only for particular CP-nets. In actual fact, results in [5] suggest it may only provide a refinement of the CP-net ordering of solutions, namely, let $\mathcal{N}$ be an acyclic $\mathrm{CP}$-net and $\succ_{\mathcal{N}}$ its induced partial preference ordering on interpretations. Then, it can be conjectured that

$$
\forall \omega, \omega^{\prime} \in \Omega, \omega \succ_{\mathcal{N}} \omega^{\prime} \Rightarrow \omega\left(\Sigma_{\mathcal{N}}\right) \succ_{\text {leximin }} \omega^{\prime}\left(\Sigma_{\mathcal{N}}\right)
$$

The following counterexample shows that the possibilistic logic representation using the leximin order may compare solutions that the CP-net leaves incomparable:

Example 1 Let us consider the CP-net of Fig. 1 with variables $V=\{X, Y, Z, S, T\}$, where $X \in\{x, \bar{x}\}$, etc., and the interpretations $\omega=x y \bar{z} \bar{s} t$ and $\omega^{\prime}=\bar{x} \bar{y} \bar{z} \bar{s} \bar{t}$. It can be checked that $\omega$ and $\omega^{\prime}$ are incomparable by the CP-net ordering, since there is no sequence of worsening flips between these two interpretations. However, the leximin order can compare them, namely $\omega \succ_{\text {leximin }} \omega^{\prime}$ : indeed $\omega\left(\Sigma_{\mathcal{N}}\right)=\left(1,1,1-\alpha_{3}, 1,1-\alpha_{5}\right)$ and 
$\omega^{\prime}\left(\Sigma_{\mathcal{N}}\right)=\left(1-\alpha_{1}, 1,1,1,1\right)$, where $1-\alpha_{1}<1-\alpha_{3}<1-\alpha_{5}$, with the convention $X=X_{1}, Y=X_{2}, Z=X_{3}, S=X_{4}$, and $T=X_{5}$.

Yet another preference relation on interpretations of a possibilistic logic base can be obtained using the symmetric Pareto ordering, denoted by $\succ_{S P}$, and defined as follows:

Definition 7 (symmetric Pareto) Let $\mathrm{v}$ and $\mathrm{v}^{\prime}$ be two vectors having the same number of components, then $\mathrm{v} \succ_{S P} \mathrm{v}^{\prime}$ if and only if there exists a permutation $\sigma$ of the components of $\mathrm{v}^{\prime}$, yielding vector $\mathrm{v}^{\prime \sigma}$, such that $\mathrm{v} \succ$ Pareto $\mathrm{v}^{\prime \sigma}$ (where as usual, $\mathrm{v} \succ$ Pareto $\mathrm{v}^{\prime}$ if and only if $\forall i, v_{i} \geq v_{i}^{\prime}$ and $\exists j, v_{j}>v_{j}^{\prime}$ ).

It is obvious that the leximin ordering refines the symmetric Pareto ordering:

$$
\mathrm{v} \succ_{S P} \mathrm{v}^{\prime} \Rightarrow \mathrm{v} \succ_{\text {leximin }} \mathrm{v}^{\prime}
$$

It was also claimed in $[7,8]$ that using the symmetric Pareto order of interpretations of the possibilistic logic encoding of a CP-net exactly captures the CP-net ordering. In fact this result seems to be true only for a special subclass of CP-nets where each node has at most one child node, (as claimed by Proposition 4 in [5]). The following counterexample shows that an exact representation of a CP-net is indeed not obtained using the symmetric Pareto order on networks where nodes can have more than one child node:

Example 2 Let us consider the CP-net of Fig. 1, together with the interpretations $\omega_{1}=$ $x y z \bar{s} \bar{t}$ and $\omega_{2}=x y \bar{z} \bar{s} \bar{t}$. We notice that $\omega_{1}$ is preferred to $\omega_{2}$ according to the CP-net order. But the symmetric Pareto order leaves them incomparable. Indeed $\omega_{1}\left(\Sigma_{\mathcal{N}}\right)=(1,1,1,1-$ $\left.\alpha_{4}, 1-\alpha_{5}\right)$ and $\omega_{2}\left(\Sigma_{\mathcal{N}}\right)=\left(1,1,1-\alpha_{3}, 1,1\right)$, with $1-\alpha_{4}<1$ and $1-\alpha_{5}<1$, while $1-\alpha_{3}<1-\alpha_{4}$ and $1-\alpha_{3}<1-\alpha_{5}$.

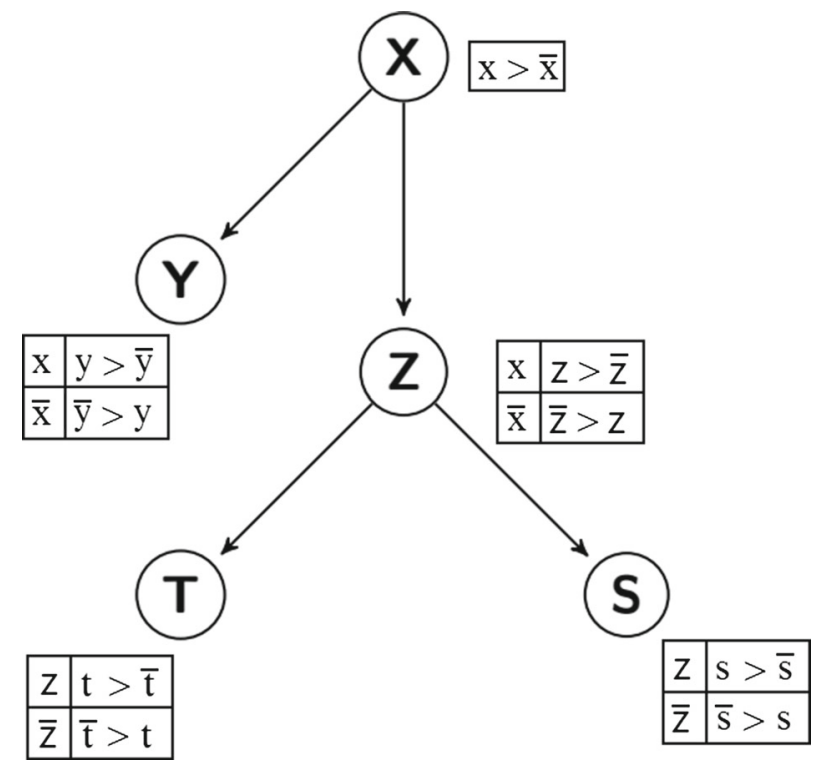

Fig. 1 CP-net associated to Example 1 
In this example, the $\mathrm{CP}$-net ordering proves more discriminant than the symmetric Pareto ordering. In contrast, the ordering $\succ_{S P}$ agrees with CP-net ordering $\succ_{\mathcal{N}}$ on the interpretations considered in Example 1, while the $\succ_{\text {leximin }}$ is in turn more discriminant than the CP-ordering.

In the general case, there are arguments to conjecture that

$$
\omega\left(\Sigma_{\mathcal{N}}\right) \succ_{S P} \omega^{\prime}\left(\Sigma_{\mathcal{N}}\right) \text { implies } \omega \succ_{\mathcal{N}} \omega^{\prime} .
$$

See [5] for a proof assuming that in a CP-net, an interpretation $\omega$ that violates more preference tables than another interpretation $\omega^{\prime}$ (in the sense of inclusion) is strictly less preferred, i.e. $\omega^{\prime} \succ_{\mathcal{N}} \omega$ (a claim that however does seem to have been proved yet).

To conclude, the question of an exact representation of any CP-net by a partially ordered set of propositional formulae remains open, but this note suggests that the discrepancies between the two representation settings look more important than expected (see [4] for additional discrepancies between CP-net and possibilistic logic, pertaining to the transitivity of priorities between father nodes and children nodes in CP-nets). All that can be expected is a formal proof that for general acyclic CP-net structures the ordering $\succ_{\mathcal{N}}$ can only be bracketed by the SP and leximin orderings induced by the associated partially ordered base $\Sigma_{\mathcal{N}}$

\section{References}

1. Boutilier, C., Brafman, R.I., Domshlak, C., Hoos, H., Poole, D.: CP-nets: A tool for representing and reasoning with conditional ceteris paribus preference statements. J. Artificial Intelligence Reasoning (JAIR) 21, 135-191 (2004)

2. Dubois, D., Kaci, S., Prade, H.: Approximation of conditional preferences networks "CP-nets" in possibilistic logic. In: Proceedings 15th IEEE Inter. Conf. on Fuzzy Systems (FUZZ-IEEE), Vancouver, July 16-21, pp. 2337-2342. IEEE (2006)

3. Dubois, D., Prade, H.: Possibilistic logic - A retrospective and prospective view. Fuzzy Sets Syst. 144, 3-23 (2004)

4. Dubois, D., Prade, H., Touazi, F.: Conditional Preference-nets, possibilistic logic, and the transitivity of priorities. In: Bramer, M., Petridis, M. (eds.) Research and Development in Intelligent Systems XXX, Proc. of AI-2013, the 33rd SGAI International Conf. on Innovative Techniques and Applications of Artificial Intelligence, Cambridge, UK, December 10-12, pp. 175-184 (2013)

5. Dubois, D., Prade, H., Touazi, F.: Conditional preference networks and possibilistic logic. In: Proceedings 12th Eur. Conf. on Symbolic and Quantitative Approaches to Reasoning with Uncertainty (ECSQARU'13), July 7-10, vol. 7958 of LNAI, pp. 181-193. Springer (2013)

6. Hadjali, A., Kaci, S., Prade, H.: Database preference queries - A possibilistic logic approach with symbolic priorities. Ann. Math. Artif. Intell. 63(3-4), 357-383 (2011)

7. Kaci, S.: Working With Preferences: Less Is More. Springer (2012)

8. Kaci, S., Prade, H.: Mastering the processing of preferences by using symbolic priorities. In: 18th European Conference on Artificial Intelligence (ECAI'08), pp. 376-380 (2008) 